\section{Padrão de consumo alimentar em mulheres no pós-parto atendidas em um centro municipal de saúde do Rio de Janeiro, Brasil}

\author{
Dietary patterns among postpartum \\ women treated at a municipal health center \\ in Rio de Janeiro, Brazil
}

Maria Beatriz Trindade de Castro ${ }^{1}$ Gilberto Kac ${ }^{2}$

Rosely Sichieri ${ }^{1}$

\section{Introdução}

This study aimed to longitudinally compare nutrient and food intakes by 276 lactating women. Dietary intake was collected using a food frequency questionnaire. Data analysis used the Student-t and ANOVA tests, comparing quantitative and qualitative dietary variation from pregnancy to postpartum. Mean daily energy intake was 2,889kcal for pregnant and 2,081 kcal for lactating women. Macronutrient dietary distributions were within internationally recommended standards. Compared to pregnancy, there was a decrease in most nutrients and foods ingested during postpartum. Dietary changes increased the density of proteins, fat, iron, rice, beans, chicken, and beef $(p<0.02)$ and decreased the density of carbohydrate, cereals, fruits, and milk and dairy products $(p<0.001)$. The results indicate a need for dietary guidelines for women, particularly during the postpartum period.

Food Consumption; Diet; Maternal Nutrition; Prenatal Nutrition
A assistência à saúde materna concentra-se basicamente na gestação 1,2, e tanto no Brasil quanto em outros países são poucas as informações sobre o estado nutricional materno na lactação 3,4,5. Adicionalmente, o retorno ao peso pré-gestacional, desejo absoluto da maioria das mães, é pouco supervisionado 6 .

$\mathrm{O}$ estado nutricional das nutrizes decorre de múltiplos determinantes, alguns dos quais refletem condições presentes antes mesmo da gravidez, como no caso do sobrepeso 7 . O efeito da lactação apresenta inúmeras vantagens para o recém-nascido e, embora tenha associado-se à redução de peso nas mulheres durante o pós-parto em alguns trabalhos 8,9, em outros estudos o efeito não foi verificado 7,10 .

O peso pré-gestacional, o ganho de peso elevado durante a gestação 5 , a velocidade da perda de peso após o parto, a duração e a intensidade da amamentação 11, a atividade física e a alimentação 11,12,13,14 são importantes preditores do estado nutricional das lactantes. Outros fatores como renda, escolaridade, estado civil, idade e paridade 5,15 também interferem nas condições maternas.

Durante a gestação, recomenda-se em média um aporte calórico extra de 300kcal. Enquanto na lactação preconiza-se um adicional de 500kcal às necessidades diárias de energia das nutrizes nos seis primeiros meses, que va- 
ria em função do estado materno pré-gestacional e do ganho de peso durante a gestação 16 . Segundo o Institute of Medicine 17, mulheres com sobrepeso (Índice de Massa Corporal IMC $\geq 26,0$ e $<29 \mathrm{~kg} / \mathrm{m}^{2}$ ) no pós-parto podem perder $2 \mathrm{~kg} / \mathrm{mês}$, no entanto, esta velocidade de perda de peso seria excessiva, após o primeiro mês do parto, para mulheres sob aleitamento exclusivo que tivessem peso pré-gestacional adequado.

No entanto, ensaio randomizado desenvolvido por McCrory et al. 13, com mulheres americanas sob aleitamento exclusivo entre o segundo e terceiro meses do pós-parto, mostrou que a perda aproximada de $1 \mathrm{~kg} /$ semana, por meio da combinação de dieta e atividade física, não interfere no crescimento da criança em mulheres que estão amamentando seus filhos exclusivamente no peito. Ainda, Lovelady et al. 14 observaram que lactantes com sobrepeso e sob aleitamento exclusivo, quando submetidas a uma restrição de $500 \mathrm{kcal}$ e à atividade física moderada, perderam em média $4,8 \mathrm{~kg}$ em dez semanas de programa e sem alteração do crescimento dos bebês.

No Brasil, poucos estudos avaliaram o consumo alimentar das mulheres no período reprodutivo e os que fizeram avaliaram gestantes adolescentes. O estudo descritivo de Barros et al. 2, com gestantes adolescentes atendidas nas maternidades do Município do Rio de Janeiro, entre julho de 1999 e março de 2001, mostrou uma inadequação do consumo de energia segundo as recomendações dietéticas do National Research Council, e que alimentos como salgados, refrigerantes, açúcar e batata frita eram freqüentes nas dietas. O estudo realizado por Azevedo \& Sampaio ${ }^{1}$ com 99 gestantes adolescentes atendidas na Maternidade Escola Assis Chateaubriand, na Cidade de Fortaleza, Ceará, mostrou uma dieta monótona, com alto teor de lipídios e inadequação de carboidratos. Mais de $60 \%$ das gestantes apresentaram uma inadequação no consumo de energia 1 . Assim sendo, este estudo teve como objetivo descrever o consumo alimentar de uma coorte de mulheres adultas no período gestacional e no pósparto, comparando os dois momentos do ciclo reprodutivo.

\section{Material e métodos}

\section{Desenho e população de estudo}

O estudo baseia-se numa coorte de mulheres no pós-parto atendidas no Centro Municipal de Saúde (CMS) Marcolino Candau, localizado no Município do Rio de Janeiro. O processo de coleta de dados ocorreu entre maio de 1999 e abril de 2001, sendo 15 meses de recrutamento e nove meses de seguimento. A população de estudo consistiu em mulheres recrutadas por meio dos serviços de pré-natal, na rotina pediátrica de imunização do bacilo de CalmetteGuerin (BCG), oferecidos pelo CMS, e imediatamente após o parto, na maternidade central de referência. O recrutamento nas consultas de pré-natal e durante a rotina de imunização do BCG foi realizado pelo pesquisador (G.K.), e na Maternidade Hospital da Praça XV por três internos devidamente treinados. Foram consideradas elegíveis: mulheres em idade reprodutiva entre 15 e 45 anos de idade, entrevistadas até trinta dias do pós-parto, sem doenças crônicas pré-existentes, com gravidez de 35 ou mais semanas gestacionais e sem gestação gemelar 5,15. Do total de 709 mulheres convidadas para participar da pesquisa, 479 aceitaram conforme fluxograma (Figura 1). Análises anteriores 15 sugerem que as perdas de seguimento foram aleatórias. Das 13 variáveis testadas, somente a idade foi estatisticamente significante. No presente estudo, foram excluídas 47 nutrizes com idade inferior a 18 anos e duas mulheres com ingestão energética superior a $6.000 \mathrm{kcal}$. Embora o protocolo excluísse mulheres com consumo inferior a $600 \mathrm{kcal}$, não houve exclusões por este motivo. Na literatura não há um ponto de corte de referência para subestimação e superestimação do consumo. Usualmente os valores adotados são, respectivamente, 500 e $5.000 \mathrm{kcal} 18$. Como o estudo aborda a dieta de lactantes, o intervalo foi deslocado para $6.000 \mathrm{kcal}$ e somente duas mulheres foram excluídas por este critério. Assim, foram consideradas elegíveis 430 mulheres para as análises de consumo alimentar descritas a seguir.

A participação no estudo foi voluntária. A pesquisa, aprovada pelo Comitê de Ética do Núcleo de Estudos em Saúde Coletiva (NESC), Universidade Federal do Rio de Janeiro (UFRJ), foi devidamente explicitada a todas as mulheres recrutadas, que assinaram um Termo de Consentimento Livre e Esclarecido.

\section{Consumo alimentar}

O consumo alimentar foi avaliado usando-se o Questionário de Freqüência de Consumo Alimentar (QFCA) validado por Sichieri \& Everhart 19. O questionário semiquantitativo utilizado para a coleta de dados dietéticos continha uma lista com 81 itens alimentares 18 . O entrevistado indicava o número de porções que costumava consumir e com que freqüência: mais 
de 3 vezes por dia; de 2 a 3 vezes por dia; 1 vez por dia; 5 a 6 vezes por semana; 2 a 4 vezes por semana; 1 vez por semana; 1 a 3 vezes por mês; nunca ou quase nunca.

O QFCA vem sendo aplicado em investigações epidemiológicas com gestantes e lactantes para medir o consumo alimentar usual 20,21,22. Estudos realizados mostram que esse instrumento é adequado para acessar o consumo de importantes nutrientes 23 e detectar mudanças dietéticas 24 na gravidez. O estudo de Barros et al. 2 utilizou um questionário de freqüência obtido com base no QFCA empregado na íntegra nesta pesquisa. A lista reduzida de alimentos era representativa da dieta deste grupo específico.

No presente estudo, o QFCA foi aplicado pelo pesquisador (G.K.) durante entrevista padronizada feita em dois momentos distintos do seguimento no pós-parto: na primeira entrevista que ocorreu até o trigésimo dia e na terceira entrevista que foi realizada no sexto mês. Assim, o tempo de referência do primeiro QFCA foi o período da gestação e o segundo os seis primeiros meses após o parto.

Dentre as 430 mulheres elegíveis, 421 (97,9\%) participantes responderam ao primeiro QFCA e $278(64,7 \%)$ ao segundo, sendo que duas mulheres que responderam ao segundo QFCA não haviam respondido ao anterior. Portanto, 276 $(64,2 \%)$ mulheres responderam ao QFCA em ambos os momentos do seguimento.

O cálculo da ingestão total de energia das participantes e da composição da dieta em cada momento do estudo foi realizado utilizando-se um programa 18 desenvolvido no pacote estatístico Statistical Analysis System - SAS versão 8.2 (SAS Institute, Cary, Estados Unidos). As porções previamente padronizadas, a partir de medidas usuais 25 , foram multiplicadas pelo número de porções consumidas e, posteriormente, por uma constante específica da freqüência indicada pela respondente, para transformar em consumo diário. Em seguida, estes valores diários obtidos eram multiplicados pelo valor da tabela de composição dos alimentos do Programa de Apoio à Nutrição elaborado pela Escola Paulista de Medicina, Universidade Federal de São Paulo 26, ou quando ausentes, da tabela de composição dos alimentos utilizada pelo Estudo Nacional de Despesa Familiar 27. Os valores dos ácidos graxos essenciais foram retirados da publicação Food Values of Portions Used 28.

\section{Análise dos dados}

As variáveis de consumo foram avaliadas quanto à sua distribuição. Uma vez que $35,8 \%$ das
Figura 1

Fluxograma da coorte no período do pós-parto.

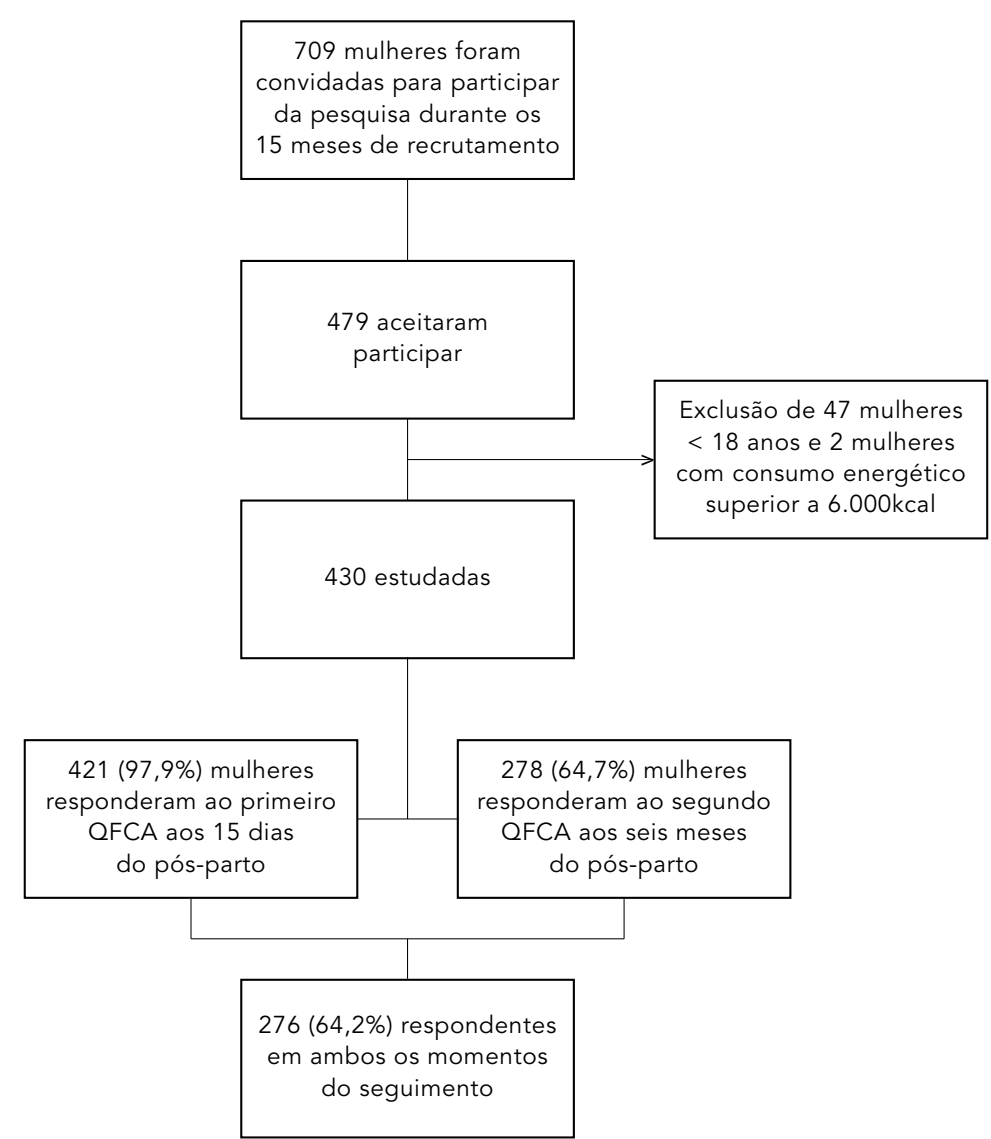

QFCA = Questionário de Freqüência de Consumo Alimentar.

participantes não responderam ao QFCA em ambos os momentos do seguimento (Figura 1), sendo nove ao primeiro e 152 ao segundo QFCA; o teste estatístico t-Student foi empregado para avaliar se o consumo entre as 145 mulheres perdidas de seguimento (não-respondentes), diferia das 276 que responderam em ambos os momentos (respondentes). Comparou-se as médias de energia, macronutrientes, micronutrientes, ácidos graxos, colesterol, alimentos ou grupos de alimentos, e da gramatura total de álcool ingerido.

As análises foram realizadas para nutrientes, alimentos e grupos de alimentos com função similar como, por exemplo, os fornecedores de energia (cereais); fornecedores de vitaminas e capacidade antioxidante (frutas e verduras) etc. Os grupos constituídos foram: (i) cereais (farinha, pão francês, pão doce, macar- 
rão, bolo, angu, aipim, pipoca, inhame, milho, batata, biscoito doce e biscoito salgado); (ii) vegetal (alface, couve, repolho, tomate, chicória, quiabo, chuchu, pepino, cebola, abóbora, abobrinha, cenoura, vagem, beterraba, couveflor e pimentão); (iii) frutas (laranja, banana, mamão, maçã, melão, abacate, abacaxi, manga, uva, goiaba e pêra); (iv) carnes (rabada, bife, carré, vísceras e lingüiça); (v) peixe (peixe, atum enlatado e camarão); (vi) leite e derivados (leite integral, leite desnatado, iogurte, queijo e requeijão); (vii) manteiga (manteiga e bacon); (viii) doces (sorvete, açúcar, caramelo, chocolate ao leite, chocolate em barra e pudim); e (ix) álcool (vinho, cerveja e bebidas alcoólicas).

As diferenças dos valores médios do consumo de energia, macronutrientes, micronutrientes, ácidos graxos, colesterol, alimentos, grupos de alimentos e álcool do período gestacional para o pós-parto foram comparadas utilizando-se para análise estatística o teste t-Student pareado.

A análise qualitativa da variação da dieta da gestação para o pós-parto foi feita de duas formas: (i) diferença da densidade de consumo de nutrientes e alimentos por $1.000 \mathrm{kcal}$ e (ii) diferença da densidade dos macronutrientes nos quartis inferior, intermediários e superior da diferença de energia. A análise de consumo a partir da densidade de nutrientes e alimentos permite conhecer a variação na composição da dieta independente do consumo energético total. O cálculo da diferença da densidade de consumo foi realizado dividindo-se os valores da gramatura/porções de macronutrientes, micronutrientes, ácidos graxos, colesterol, alimentos e álcool pelo consumo energético total em ambos os momentos do seguimento. Empregou-se o teste t-Student pareado para verificar a diferença das médias da densidade nestes períodos. Enquanto a análise dos quartis da diferença de energia (energia na gestação energia do pós-parto) pela diferença da densidade dos macronutrientes da dieta (densidade dos macronutrientes da gestação - densidade dos macronutrientes do pós-parto) avaliou a variação da composição da dieta entre as participantes que mais restringiram o consumo alimentar, daquelas com menor restrição. Um resultado positivo indicava que o valor consumido era maior no período gestacional e, ao contrário, quando negativo. Optou-se pelo teste estatístico ANOVA para verificar as diferenças da densidade de consumo dos macronutrientes nos quartis da diferença de energia.

\section{Resultados}

Na linha de base, o tempo médio de pós-parto foi de 16 dias com desvio-padrão (DP) de 7,27 dias, e a média de idade foi de 25 anos com DP de 6,14 anos. A maior parte das mulheres da coorte era casada ou vivia sob união estável e $57,2 \%$ já haviam tido pelo menos uma gestação anterior. Reportaram ser fumantes $14,1 \%$ das entrevistadas (Tabela 1).

Embora as perdas de seguimento somem $35,8 \%$, somente foram estatisticamente significantes as diferenças relativas ao consumo de cerveja $(\mathrm{p}=0,02)$ e álcool $(\mathrm{p}=0,02)$ (Tabela 2). As mulheres que não foram incluídas nas análises consumiam estes alimentos em maiores quantidades.

As mulheres reportaram um consumo energético médio de $2.889 \mathrm{kcal}$ durante a gestação e de $2.081 \mathrm{kcal}$ no pós-parto (Tabela 3). A diferença média do consumo energético de $808 \mathrm{kcal}$ com DP de 839kcal, não apresentada na tabela, equivale aproximadamente a uma diferença média de 23g de proteínas com DP de 26g; $141 \mathrm{~g}$ de carboidratos e DP de 152g; e 20g de lipídios com DP de 27g. Os valores percentuais dos macronutrientes da dieta durante a gestação foram de $11,9 \%$ de proteína, $65 \%$ de carboidratos e $23 \%$ de lipídios e no pós-parto foram respectivamente, $12,3 \%, 64 \%$ e $23,4 \%$.

No presente estudo, 21 (7,6\%) gestantes relataram um consumo abaixo de $1.800 \mathrm{kcal}$, sendo três $(1,01 \%)$ abaixo de $1.500 \mathrm{kcal}$. Entre as lactantes, $96(34,8 \%)$ reportaram um consumo inferior a $1.800 \mathrm{kcal}$, sendo que destas, $50(52,1 \%)$ tiverem um consumo inferior a $1.500 \mathrm{kcal}$. Cerca de $60 \%$ das mulheres restringiram o consumo de energia em mais de $20 \%$ no pós-parto, sendo que $40 \%$ delas restringiram o consumo em mais de $40 \%$. Somente 37 mães $(13,4 \%)$ aumentaram o consumo no pós-parto.

Com exceção do café $(p=0,01)$, vinho $(p=$ $0,14)$, cerveja $(p=0,03)$ e álcool $(p=0,03)$, que apresentaram um incremento na dieta do pósparto, todos os demais valores obtidos de consumo: energia, macronutrientes, micronutrientes, ácidos graxos e colesterol apresentam uma queda importante e estatisticamente significante entre os dois períodos do ciclo reprodutivo (Tabela 3). Nota-se, por exemplo, que na gestação o número de porções consumidas de frutas, leite desnatado e salgados era praticamente o dobro do período do pós-parto. As porções de cereais, leite integral, leite e derivados também foram bastante reduzidas no pósparto.

A diferença de densidade de alimentos permitiu qualificar a variação da composição da 
dieta durante o ciclo reprodutivo. Houve um decréscimo na densidade de carboidratos e um aumento de proteínas e lipídios (Tabela 4). Para cada $1.000 \mathrm{kcal}$ consumidas houve uma diminuição de 3,4g de carboidratos com DP de $17,6 \mathrm{~g}$ e um incremento de $0,7 \mathrm{~g}$ de proteína com DP de 4,9g, e 0,5g de lipídios com DP de 6,49g (dados não apresentados na tabela). Entre os micronutrientes, diminuiu a densidade do consumo de cálcio e vitamina C. A dieta ficou mais rica em ferro e o decréscimo de vitamina A não foi estatisticamente significativo $(p=0,21)$. A diferença da densidade do consumo de vegetais $(p=0,10)$ e manteiga $(p=0,85)$, alimentos ricos em vitamina $A$, também não mostrou diferenças significantes. Com relação às gorduras, houve um incremento na densidade do consumo de ácidos graxos saturados (Tabela 4).

A dieta das mulheres no pós-parto apresentou uma menor densidade do consumo de cereais, frutas, leite e derivados, leite integral e leite desnatado, e um aumento qualitativo do consumo de arroz, feijão, macarrão, carnes, frango, doces, café, vinho e cerveja. Os refrigerantes, salgados e bebidas alcoólicas não tiveram seu padrão qualitativo alterado $(\mathrm{p}>0,17)$ (Tabela 4).

As mulheres que tiveram a menor diferença do valor energético da dieta da gestação para o pós-parto (quartil inferior) passaram a consumir uma dieta com menor densidade de proteínas ( $\mathrm{p}=0,05)$ e lipídios, e maior densidade de carboidratos $(p=0,05)$ quando comparadas com os quartis intermediários. As mulheres que tiveram uma diferença maior na quantidade de energia (quartil superior) consumida da gestação para o pós-parto diminuíram a densidade de carboidratos e aumentaram a densidade de proteínas $(\mathrm{p}=0,05)$ e lipídios da dieta quando comparadas com o quartil inferior.

\section{Discussão}

As mulheres investigadas apresentaram uma alta ingestão de energia no período gestacional e uma queda acentuada do consumo de quase todos os alimentos e nutrientes no período do pós-parto, sendo que $40 \%$ restringiram o consumo em mais de $40 \%$. A subestimação no consumo alimentar 19,29 poderia justificar este decréscimo do consumo em um período de alta demanda de energia. Contudo, outros estudos mostraram importante redução das calorias consumidas no pós-parto. Assim, o estudo longitudinal de George et al. 22, realizado com mulheres americanas, encontrou um decréscimo no consumo energético da gravidez para o pós-
Tabela 1

Características demográficas, sócio-econômicas e antropométricas na linha de base das 276 mulheres respondentes do Questionário de Freqüência de Consumo Alimentar (QFCA) no pós-parto. Rio de Janeiro, Brasil, 1999/2001.

\begin{tabular}{|c|c|c|}
\hline Variáveis & $\mathbf{n}$ & Freqüência (\%) \\
\hline \multicolumn{3}{|l|}{ Idade (anos) } \\
\hline $18-29$ & 204 & 73,9 \\
\hline$\geq 30$ & 72 & 26,1 \\
\hline \multicolumn{3}{|l|}{ Estado civil } \\
\hline Casada/Em união & 219 & 79,3 \\
\hline Solteira & 57 & 20,7 \\
\hline \multicolumn{3}{|l|}{ Paridade } \\
\hline 1 & 118 & 42,8 \\
\hline$\geq 2$ & 158 & 57,2 \\
\hline \multicolumn{3}{|c|}{ Índice de Massa Corporal (kg/m²) } \\
\hline$<18,5$ & 9 & 3,2 \\
\hline $18,5-24,9$ & 141 & 51,1 \\
\hline $25,0-29,9$ & 91 & 33,0 \\
\hline$\geq 30,0$ & 35 & 12,7 \\
\hline \multicolumn{3}{|l|}{ Atividade de lazer } \\
\hline Sim & 161 & 58,3 \\
\hline Não & 115 & 41,7 \\
\hline \multicolumn{3}{|l|}{ Cor da pele } \\
\hline Branca & 104 & 37,7 \\
\hline Parda & 125 & 45,3 \\
\hline Negra & 47 & 17,0 \\
\hline \multicolumn{3}{|l|}{ Tabagismo } \\
\hline Fumantes & 39 & 14,1 \\
\hline Não-fumantes & 237 & 85,9 \\
\hline \multicolumn{3}{|c|}{ Renda familiar total (salários mínimos)* } \\
\hline$\leq 1$ & 44 & 15,9 \\
\hline $1-3$ & 145 & 52,5 \\
\hline $4-5$ & 57 & 20,7 \\
\hline$>5$ & 30 & 10,9 \\
\hline \multicolumn{3}{|c|}{ Anos de escolaridade } \\
\hline$\leq 4$ & 72 & 26,1 \\
\hline $5-8$ & 116 & 42,0 \\
\hline$\geq 9$ & 88 & 31,9 \\
\hline
\end{tabular}

* Salário mínimo em março de 1999: R\$130,00.

parto de 2.571 para $2.160 \mathrm{kcal}$, respectivamente. O ensaio clínico realizado por Krebs et al. 30 encontrou um declínio significativo no consumo de energia entre as lactantes americanas ao longo dos sete meses de seguimento. O estudo longitudinal realizado por Mackey et al. 4 , também com mulheres americanas, mostrou uma 
Média e desvio-padrão (DP) do consumo das mulheres perdidas de seguimento e as diferenças das médias entre as 145 mulheres perdidas e as 276 não perdidas de seguimento. Rio de Janeiro, Brasil, 1999/2001.

\begin{tabular}{|c|c|c|c|c|}
\hline \multirow[t]{2}{*}{ Variáveis } & \multicolumn{4}{|c|}{ Perdas de seguimento } \\
\hline & Consumo médio & DP & Diferença das médias & Valor de $\mathrm{p}^{*}$ \\
\hline \multicolumn{5}{|l|}{ Macronutrientes } \\
\hline Energia (kcal) & 2.870 & 884 & -19 & 0,82 \\
\hline Proteína (g) & 87,0 & 30,2 & $-0,6$ & 0,83 \\
\hline Carboidrato (g) & 470 & 144 & $-6,8$ & 0,65 \\
\hline Lipídio (g) & 75,0 & 30,3 & 0,01 & 0,99 \\
\hline \multicolumn{5}{|l|}{ Micronutrientes } \\
\hline Cálcio (mg) & 787 & 332 & 12,3 & 0,70 \\
\hline Ferro $(\mathrm{mg})$ & 16,0 & 5,35 & $-0,50$ & 0,32 \\
\hline Vitamina A (UI) & 2.926 & 2.304 & -135 & 0,54 \\
\hline Vitamina C (mg) & 395 & 261 & $-5,5$ & 0,84 \\
\hline \multicolumn{5}{|l|}{ Ácidos graxos } \\
\hline Saturado (g) & 24,4 & 10,5 & 0,4 & 0,65 \\
\hline Polinsaturado (g) & 10,6 & 5,78 & $-0,5$ & 0,44 \\
\hline Monoinsaturado (g) & 7,2 & 4,21 & 0,6 & 0,14 \\
\hline Colesterol (mg) & 275 & 156 & 11,4 & 0,45 \\
\hline \multicolumn{5}{|l|}{ Alimentos } \\
\hline Arroz (colher sopa cheia) & 8,47 & 5,49 & $-0,13$ & 0,80 \\
\hline Feijão (concha média) & 1,92 & 1,29 & $-0,16$ & 0,20 \\
\hline Macarrão (escumadeira) & 0,55 & 0,53 & 0,01 & 0,79 \\
\hline Cereal (porção) & 8,24 & 5,38 & 0,24 & 0,67 \\
\hline Vegetal (colher sopa cheia) & 7,91 & 5,96 & $-0,07$ & 0,90 \\
\hline Frutas (unidade) & 3,82 & 2,89 & $-0,18$ & 0,57 \\
\hline Ovo (unidade) & 0,35 & 0,42 & 0,05 & 0,27 \\
\hline Carnes (pedaço médio) & 0,87 & 0,55 & $-0,01$ & 0,94 \\
\hline Frango (pedaço médio) & 0,68 & 0,70 & $-0,04$ & 0,54 \\
\hline Peixe (posta) & 0,22 & 0,31 & $-0,04$ & 0,40 \\
\hline Leite integral (copo) & 1,13 & 1,03 & $-0,02$ & 0,88 \\
\hline Leite desnatado (copo) & 0,12 & 0,41 & $-0,05$ & 0,29 \\
\hline Leite e derivados (porção) & 2,12 & 1,78 & 0,06 & 0,75 \\
\hline Salgados (unidade) & 0,29 & 0,59 & 0,10 & 0,06 \\
\hline Manteiga (freqüência) & 1,50 & 1,05 & 0,06 & 0,54 \\
\hline Doces (fatia ou barra) & 7,46 & 5,51 & 0,22 & 0,69 \\
\hline Refrigerante (copo) & 1,31 & 1,64 & 0,13 & 0,43 \\
\hline Café (cafezinho) & 1,06 & 0,98 & 0,11 & 0,22 \\
\hline Suco (copo) & 1,38 & 1,24 & 0,02 & 0,85 \\
\hline Vinho (taça) & 0,03 & 0,19 & 0,02 & 0,44 \\
\hline Cerveja (copo) & 0,38 & 1,30 & 0,28 & 0,02 \\
\hline Bebidas alcoólicas (dose) & 0,004 & 0,03 & 0,002 & 0,46 \\
\hline Álcool (g) & 5,32 & 17,92 & 3,79 & 0,02 \\
\hline
\end{tabular}

UI = unidade internacional.

* Teste t-Student. 
Média e desvio-padrão (DP) dos valores de consumo alimentar de 276 mulheres nos períodos gestacional e pós-parto em uma coorte com nove meses de seguimento. Rio de Janeiro, Brasil, 1999/2001.

\begin{tabular}{|c|c|c|c|c|c|}
\hline \multirow[t]{2}{*}{ Variáveis } & \multicolumn{2}{|c|}{ Gestacional } & \multicolumn{2}{|c|}{ Pós-parto } & \multirow[t]{2}{*}{ Valor de $p^{\star \star}$} \\
\hline & Média* & $D P^{*}$ & Média & DP & \\
\hline \multicolumn{6}{|l|}{ Macronutrientes } \\
\hline Energia (kcal) & 2.889 & 824 & 2.081 & 676 & $<0,0001$ \\
\hline Proteína (g) & 87,6 & 26,0 & 64,5 & 21,4 & $<0,0001$ \\
\hline Carboidrato (g) & 477 & 142 & 336 & 113 & $<0,0001$ \\
\hline Lipídio (g) & 75,0 & 28,8 & 55,2 & 24,0 & $<0,0001$ \\
\hline \multicolumn{6}{|l|}{ Micronutrientes } \\
\hline Cálcio (mg) & 774 & 280 & 527 & 235 & $<0,0001$ \\
\hline Ferro (mg) & 16,5 & 4,68 & 12,6 & 4,09 & $<0,0001$ \\
\hline Vitamina A (UI) & 3.061 & 2.021 & 2101 & 1336 & $<0,0001$ \\
\hline Vitamina C (mg) & 401 & 260 & 233 & 189 & $<0,0001$ \\
\hline \multicolumn{6}{|l|}{ Ácidos graxos } \\
\hline Saturado (g) & 24,0 & 9,42 & 18,2 & 8,29 & $<0,0001$ \\
\hline Polinsaturado (g) & 11,1 & 6,11 & 7,9 & 4,50 & $<0,0001$ \\
\hline Monoinsaturado (g) & 6,6 & 3,29 & 4,9 & 2,97 & $<0,0001$ \\
\hline Colesterol (mg) & 264 & 138 & 196 & 103 & $<0,0001$ \\
\hline \multicolumn{6}{|l|}{ Alimentos } \\
\hline Arroz (colher sopa cheia) & 8,60 & 4,17 & 7,81 & 4,07 & 0,0043 \\
\hline Feijão (concha média) & 2,08 & 1,19 & 1,69 & 1,03 & $<0,0001$ \\
\hline Macarrão (escumadeira) & 0,54 & 0,54 & 0,46 & 0,47 & 0,0143 \\
\hline Cereal (porção) & 8,00 & 5,58 & 4,55 & 3,46 & $<0,0001$ \\
\hline Vegetal (colher sopa cheia) & 7,98 & 4,99 & 6,14 & 3,81 & $<0,0001$ \\
\hline Frutas (unidade) & 4,00 & 3,15 & 1,94 & 1,86 & $<0,0001$ \\
\hline Ovo (unidade) & 0,30 & 0,34 & 0,24 & 0,26 & 0,0057 \\
\hline Carnes (pedaço médio) & 0,88 & 0,56 & 0,75 & 0,54 & 0,0009 \\
\hline Frango (pedaço médio) & 0,72 & 0,62 & 0,56 & 0,39 & $<0,0001$ \\
\hline Peixe (posta) & 0,26 & 0,53 & 0,19 & 0,29 & 0,0479 \\
\hline Leite integral (copo) & 1,15 & 1,01 & 0,68 & 0,74 & $<0,0001$ \\
\hline Leite desnatado (copo) & 0,17 & 0,51 & 0,07 & 0,31 & $<0,0017$ \\
\hline Leite e derivados (porção) & 2,06 & 1,46 & 1,27 & 1,11 & $<0,0001$ \\
\hline Salgados (unidade) & 0,19 & 0,38 & 0,10 & 0,28 & 0,0001 \\
\hline Manteiga (freqüência) & 1,44 & 0,88 & 1,07 & 0,81 & $<0,0001$ \\
\hline Doces (fatia ou barra) & 7,24 & 5,28 & 5,97 & 4,53 & 0,0003 \\
\hline Refrigerante (copo) & 1,18 & 1,68 & 0,78 & 1,11 & 0,0001 \\
\hline Café (cafezinho) & 0,95 & 0,90 & 1,09 & 0,95 & 0,0112 \\
\hline Suco (copo) & 1,36 & 1,21 & 0,88 & 0,90 & $<0,0001$ \\
\hline Vinho (taça) & 0,01 & 0,11 & 0,02 & 0,09 & 0,1436 \\
\hline Cerveja (copo) & 0,10 & 0,48 & 0,19 & 0,62 & 0,0346 \\
\hline Bebidas alcoólicas (dose) & 0,0016 & 0,03 & 0,0016 & 0,03 & 1,0000 \\
\hline Álcool (g) & 1,53 & 6,85 & 2,80 & 8,64 & 0,0250 \\
\hline
\end{tabular}

$\mathrm{UI}=$ unidade internacional.

* Valores das médias e DP das respondentes.

** Teste t-Student pareado. 
Média e desvio-padrão (DP) dos valores da densidade do consumo por $1.000 \mathrm{kcal}$ de 276 mulheres nos períodos gestacional e pós-parto em uma coorte com nove meses de seguimento. Rio de Janeiro, Brasil, 1999/2001.

\begin{tabular}{|c|c|c|c|c|c|}
\hline \multirow[t]{2}{*}{ Variáveis } & \multicolumn{2}{|c|}{ Gestacional } & \multicolumn{2}{|c|}{ Pós-parto } & \multirow[t]{2}{*}{ Valor de $p^{\star}$} \\
\hline & Média & DP & Média & DP & \\
\hline \multicolumn{6}{|l|}{ Macronutrientes } \\
\hline Proteína (g) & 30,6 & 4,17 & 31,3 & 4,45 & 0,0173 \\
\hline Carboidrato (g) & 165,2 & 14,99 & 161,8 & 15,85 & 0,0014 \\
\hline Lipídio (g) & 25,8 & 5,69 & 26,3 & 6,02 & 0,1653 \\
\hline \multicolumn{6}{|l|}{ Micronutrientes } \\
\hline Cálcio (mg) & 268 & 63,6 & 251 & 66,4 & $<0,0001$ \\
\hline Ferro (mg) & 5,8 & 1,13 & 6,1 & 1,23 & $<0,0001$ \\
\hline Vitamina A (UI) & 1080 & 679 & 1019 & 564 & 0,2134 \\
\hline Vitamina C (mg) & 137 & 74,9 & 109 & 68,9 & $<0,0001$ \\
\hline \multicolumn{6}{|l|}{ Ácidos graxos } \\
\hline Saturado (g) & 8,3 & 2,33 & 8,7 & 2,34 & 0,0136 \\
\hline Polinsaturado (g) & 3,8 & 1,74 & 3,8 & 1,59 & 0,6907 \\
\hline Monoinsaturado (g) & 2,3 & 1,02 & 2,3 & 1,10 & 0,8023 \\
\hline Colesterol (mg) & 92,1 & 43,8 & 94,6 & 41,6 & 0,4234 \\
\hline \multicolumn{6}{|l|}{ Alimentos } \\
\hline Arroz (colher sopa cheia) & 3,10 & 1,54 & 3,93 & 2,15 & $<0,0001$ \\
\hline Feijão (concha média) & 0,76 & 0,47 & 0,84 & 0,50 & 0,0097 \\
\hline Macarrão (escumadeira) & 0,19 & 0,19 & 0,22 & 0,21 & 0,0306 \\
\hline Cereal (porção) & 2,71 & 1,62 & 2,14 & 1,34 & $<0,0001$ \\
\hline Vegetal (colher sopa cheia) & 2,91 & 1,94 & 3,12 & 1,98 & 0,1034 \\
\hline Frutas (unidade) & 1,36 & 0,90 & 0,91 & 0,74 & $<0,0001$ \\
\hline Ovo (unidade) & 0,11 & 0,13 & 0,12 & 0,13 & 0,2653 \\
\hline Carnes (pedaço médio) & 0,31 & 0,18 & 0,36 & 0,21 & 0,0002 \\
\hline Frango (pedaço médio) & 0,26 & 0,20 & 0,29 & 0,24 & 0,0129 \\
\hline Peixe (posta) & 0,09 & 0,16 & 0,09 & 0,13 & 0,9526 \\
\hline Leite integral (copo) & 0,42 & 0,38 & 0,35 & 0,40 & 0,0100 \\
\hline Leite desnatado (copo) & 0,07 & 0,22 & 0,04 & 0,16 & 0,0088 \\
\hline Leite e derivados (porção) & 0,75 & 0,56 & 0,62 & 0,54 & 0,0002 \\
\hline Salgados (unidade) & 0,06 & 0,11 & 0,05 & 0,11 & 0,1733 \\
\hline Manteiga (freqüência) & 0,51 & 0,32 & 0,51 & 0,38 & 0,8461 \\
\hline Doces (fatia ou barra) & 2,51 & 1,75 & 2,85 & 1,94 & 0,0106 \\
\hline Refrigerante (copo) & 0,39 & 0,52 & 0,36 & 0,48 & 0,3595 \\
\hline Café (cafezinho) & 0,36 & 0,36 & 0,55 & 0,50 & $<0,0001$ \\
\hline Suco (copo) & 0,47 & 0,38 & 0,42 & 0,39 & $<0,1249$ \\
\hline Vinho (taça) & 0,004 & 0,03 & 0,012 & 0,05 & 0,0113 \\
\hline Cerveja (copo) & 0,04 & 0,19 & 0,09 & 0,28 & 0,0035 \\
\hline Bebidas alcoólicas (dose) & 0,0006 & 0,01 & 0,0010 & 0,02 & 0,7590 \\
\hline Álcool (g) & 0,6 & 2,69 & 1,3 & 3,98 & 0,0017 \\
\hline
\end{tabular}

$\mathrm{UI}=$ unidade internacional.

* Teste t-Student pareado. 
Diferença média de densidade de consumo de proteínas, carboidratos e lipídios por 1.000kcal entre as mulheres com menores, intermediárias e maiores variações da diferença no consumo total de energia em uma coorte de nove meses de seguimento. Rio de Janeiro, Brasil, 1999/2001.

\begin{tabular}{|c|c|c|c|c|c|}
\hline Variação no consumo de energia & $\mathbf{n}$ & Média & DP & Mínimo & Máximo \\
\hline \multicolumn{6}{|l|}{ Quartil inferior } \\
\hline Proteínas & 69 & 1,3 & 4,37 & $-7,7$ & $9,9 *, * *, * * *$ \\
\hline Carboidratos & 69 & $-1,5$ & 16,53 & $-40,9$ & $51,2^{\star, \star \star}$ \\
\hline Lipídios & 69 & 0,6 & 6,50 & $-19,8$ & 17,1 \\
\hline \multicolumn{6}{|l|}{ Quartis intermediários } \\
\hline Proteínas & 138 & $-1,0$ & 4,54 & $-12,1$ & $11,0 *, \star \star$ \\
\hline Carboidratos & 138 & 5,1 & 17,81 & $-55,1$ & $49,1 *, \star \star$ \\
\hline Lipídios & 138 & $-1,0$ & 6,51 & $-14,5$ & 24,5 \\
\hline \multicolumn{6}{|l|}{ Quartil superior } \\
\hline Proteínas & 69 & $-2,1$ & 5,43 & $-13,9$ & 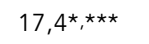 \\
\hline Carboidratos & 69 & 5,0 & 17,63 & $-35,1$ & 52,9 \\
\hline Lipídios & 69 & $-0,7$ & 6,40 & $-17,8$ & 13,6 \\
\hline
\end{tabular}

* Teste Scheffe's.

** Valor de $\mathrm{p}<0,05$ entre a diferença da densidade de consumo dos quartis inferior e intermediário.

$\star * *$ Valor de $\mathrm{p}<0,05$ entre a diferença da densidade de consumo dos quartis inferior e superior.

diminuição do consumo de energia do terceiro para o sexto mês do pós-parto, respectivamente de 2.278 para $2.165 \mathrm{kcal}$.

O QFCA pode subestimar ou superestimar o consumo de alimentos em função dos itens acrescentados na lista de freqüência 31 . Na avaliação comparativa de dois períodos próximos no tempo é pouco provável que haja mudanças na acurácia do instrumento. As alterações do período gravídico para o pós-parto parecem expressar alterações reais de consumo. Convém lembrar, que este instrumento tem sido o mais utilizado na aferição de consumo usual, sendo estável no tempo ${ }^{31}$.

Restrição energética não tem sido recomendada durante a gravidez, nem no caso de mulheres obesas. No pós-parto, preconiza-se que dieta restritiva somente deveria ocorrer quando a lactação estivesse estabilizada, nunca no início da amamentação ${ }^{32}$. O Institute of Medicine 17 recomenda um consumo de no mínimo $1.800 \mathrm{kcal}$ para assegurar os nutrientes da dieta para a mãe e para o bebê amamentado no peito, e adverte sobre a prática de dietas com valor energético inferior a $1.500 \mathrm{kcal}$, dietas líquidas e o uso de medicamentos para a perda de peso durante a lactação.

No presente estudo, relataram um consumo abaixo de $1.800 \mathrm{kcal} 7,6 \%$ das gestantes e $34,8 \%$ das lactantes, sendo que entre as nutrizes, $52,1 \%$ tiveram um consumo inferior a $1.500 \mathrm{kcal}$ e me- nos de $40 \%$ alcançou um consumo de $2.200 \mathrm{kcal}$.

Além da diminuição quantitativa do consumo da gestação para o pós-parto, houve uma variação na composição da dieta. Nutrientes que são de fato importantes para o estado nutricional da mulher na lactação, tiveram as suas densidades de consumo diminuídas: cálcio e vitamina C. A redução do consumo de produtos lácteos e frutas pode ter contribuído, respectivamente, para o decréscimo desses nutrientes. Assim, as recomendações do Dietary Guidelines Advisory Committee 33 para mulheres ativas com uma dieta de $2.200 \mathrm{kcal}$, de duas a três porções de leite e derivados, e três porções de frutas, que encontravam-se limítrofes na gestação, foram reduzidas em mais ou menos $50 \%$ no pós-parto.

A densidade do consumo de macarrão aumentou no pós-parto, embora tenha sido observado um decréscimo no consumo de cereais, que pode ser decorrente de menor ingestão de pães salgados e doces, biscoitos, bolos, tubérculos ou raízes. Assim como o frango, a densidade de carnes aumentou na lactação e estes aumentos qualitativos parecem ter refletido na maior densidade de ferro.

A dieta ficou mais rica em proteínas e gorduras saturadas. Houve uma elevação na densidade de proteínas e lipídios da dieta e diminuição na densidade dos carboidratos. Essas alterações da composição da dieta ficam bas- 
tante nítidas quando se avalia a variação da diferença da densidade dos macronutrientes nos quartis pela diferença de energia. Mackey et al. 4 também encontraram uma diferença da variação das densidades dos macronutrientes da dieta dos três meses para os seis meses do pósparto em mulheres americanas.

Mesmo com as variações no pós-parto, os percentuais dos macronutrientes encontraram-se dentro dos intervalos preconizados pela Organização Mundial da Saúde 34 de 10 a $15 \%$ de proteínas; 50 a $70 \%$ de carboidratos; e 15 a 30\% de lipídios, embora o limite de gordura seja o limite máximo e não o recomendado. Ainda, ressalva-se que os valores percentuais de lipídios podem estar subestimados, visto que alimentos como a manteiga, margarina e bacon são computados multiplicando-se a freqüência de consumo indicada pelo indivíduo por uma porção previamente padronizada, não discriminando indivíduos que consomem grandes porções de gorduras, os que utilizam óleos e banhas em demasia no preparo de alimentos ou os que adicionam azeite ou óleos à comida preparada ou em saladas.

As informações dietéticas coletadas no estudo realizado por Doran \& Evers 3 , com mulheres canadenses de baixa renda, aos três meses do pós-parto, mostraram um consumo percentual de gordura de $30 \%$. Boardley et al. 20 encontraram dietas com percentuais de lipídios que variavam de 37,5 para $40,7 \%$, respectivamente, em mulheres americanas brancas e negras assistidas no programa Special Supplemental Food Program for Women, Infants and Children.

Apesar das orientações durante a lactação 17,35 desencorajarem o uso de bebidas alcoólicas e que contenham cafeína, as mães do nosso estudo aumentaram o consumo destes alimentos na lactação, embora em quantidades mínimas. Do período gestacional para o pósparto dobrou o consumo de cerveja, vinho e álcool e aumentou em $15 \%$ a ingestão de café. A densidade do consumo de vinho triplicou e o de café aumentou em 50\%. O baixo consumo de café observado entre as mulheres durante a gestação parece concordar com os resultados obtidos no estudo de caso-controle realizado por Souza 36, na maternidade do Instituto Fernandes Figueira, Fundação Oswaldo Cruz, que mostrou que alimentos cafeinados são consumidos em quantidades muito baixas pelas brasileiras.

O consumo em gramas de peixe, ovo, manteiga, salgados, sucos e refrigerantes declinou no pós-parto, mas suas densidades não foram alteradas significativamente. Parece que estes alimentos têm um padrão de consumo usual fixo.

Alimentos tradicionais da dieta do brasileiro, o arroz e o feijão, que têm perdido espaço para alimentos industrializados e de rápido preparo 37 , apresentaram um incremento das suas densidades na composição da dieta no pós-parto. Se o Institute of Medicine 17 afirma que nos primeiros seis meses do pós-parto o apetite é o melhor guia para saber o quanto se deve comer, por que não buscar orientar a dieta durante a lactação com o consumo de alimentos saudáveis, ricos em nutrientes e típicos da culinária nacional? No Brasil, dois estudos mostraram que o padrão dietético baseado no consumo de arroz e feijão é protetor e não está associado ao excesso de peso 38,39.

Enfim, além de escassos os estudos realizados durante o pós-parto que tenham como foco o estado nutricional da mulher e, mais especificamente, o seu consumo de alimentos, as recomendações durante a lactação centram-se basicamente no aleitamento materno sem focar a atenção na saúde da mulher. No presente estudo, embora aspectos positivos tenham sido observados, como o aumento qualitativo no consumo de arroz e feijão, frango e carne, o que possibilitou uma dieta mais rica em ferro, percebeu-se claramente a necessidade de orientar as mães durante todo o período do ciclo reprodutivo. As mulheres modificaram suas dietas, contudo sem adequá-las para uma alimentação saudável. 


\section{Resumo}

O presente estudo comparou longitudinalmente o consumo de nutrientes e alimentos em 276 mulheres atendidas em um centro municipal de saúde do Rio de Janeiro, Brasil, no pós-parto. As informações dietéticas foram obtidas com base no Questionário de Freqüência de Consumo Alimentar. Analisou-se a diferença quantitativa e a variação qualitativa da composição da dieta do período gestacional para o pós-parto. Os testes estatísticos empregados foram $t$-Student pareado e ANOVA. O consumo energético médio foi de 2.889kcal na gestação e 2.081 kcal no pós-parto. Nos dois momentos avaliados, o consumo de macronutrientes encontrava-se dentro dos valores recomendados, com declínio significativo $(p<0,05)$ da maioria dos nutrientes e alimentos consumidos no pós-parto. Quanto à composição da dieta, observou-se um incremento na densidade de proteínas, gorduras saturadas, ferro, arroz, feijão, frango e carne $(p<0,02)$ e diminuição da densidade de carboidratos, cereais, frutas, leite e derivados ( $p<0,001)$. Os resultados indicam a necessidade de orientação nutricional, particularmente no período do pós-parto, visto que muitas mudanças na dieta foram inadequadas.

Consumo de Alimentos; Dieta; Nutrição Materna; Nutrição Pré-natal

\section{Colaboradores}

G. Kac planejou a pesquisa e coletou os dados. M. B. T. Castro e R. Sichieri realizaram as análises. Todos os autores participaram das discussões sobre os resultados e da preparação da versão final do texto.

\section{Agradecimentos}

À Coordenação de Aperfeiçoamento de Pessoal de Nível Superior (CAPES) pela bolsa de doutorado concedida à M. B. T. Castro e G. Kac. O projeto de pesquisa original foi financiado pelas seguintes fontes: Fundação Universitária José Bonifácio da Universidade Federal do Rio de Janeiro e Fundação Carlos Chagas Filho de Amparo à Pesquisa do Estado do Rio de Janeiro. G. Kac é Pesquisador II do Conselho Nacional de Desenvolvimento Científico e Tecnológico.

\section{Referências}

1. Azevedo DV, Sampaio HAC. Consumo alimentar de gestantes adolescentes atendidas em serviços de assistência pré-natal. Rev Nutr 2003; 16:27380 .

2. Barros DC, Pereira RA, Gama SGN, Leal MC. O consumo alimentar de gestantes adolescentes no Município do Rio de Janeiro. Cad Saúde Pública 2004; 20 Suppl 1:S121-9.

3. Doran L, Evers S. Energy and nutrient inadequacies in the diets of low-income women who breastfeed. J Am Diet Assoc 1997; 97:1283-7.

4. Mackey AD, Picciano MF, Mitchell DC, SmiciklasWright H. Self-selected diets of lactating women often fail to meet dietary recommendations. J Am Diet Assoc 1998; 98:297-302.

5. Kac G, Benício MHD, Meléndez GV, Valente JG. Nine months postpartum weight retention predictors for Brazilian women. Public Health Nutr 2004; 75:621-8.

6. Dewey KG. Effects of caloric restriction and exercise during lactation. J Nutr 1998; 128:386S-95S.

7. Sichieri R, Field AE, Rich-Edwards J, Willet WC. Prospective assessment of exclusive breastfeeding in relation to weight change in women. Int J Obes 2003; 27:815-20.

8. Janney CA, Zhang D, Sowers M. Lactation and weight retention. Am J Clin Nutr 1997; 69:583-4.

9. Kac G. Fatores determinantes da retenção de peso no pós-parto em uma coorte de mulheres com 9 meses de seguimento [Tese de Doutorado]. São Paulo: Faculdade de Saúde Pública, Universidade de São Paulo; 2002.

10. Coitinho DC, Sichieri R, Benício MHD. Obesity and weight change related to parity and breastfeeding among parous women in Brazil. Public Health Nutr 2001; 4:865-70.

11. Organización Mundial de la Salud. El estado físico: uso e interpretación de la antropometría. Geneva: Organización Mundial de la Salud; 1995. (Serie de Informes Técnicos, 854).

12. Dewey KG, Lovelady CA, Nommsen-Rivers LA, McCrory MA, Lönnerdal B. A randomized study of the effects of aerobic exercise by lactating women on breast-milk volume and composition. N Engl J Med 1994; 330:449-53.

13. McCrory MA, Nommsen-Rivers LA, Molé PA, Lönnerdal B, Dewey KG. Randomized trial of the short-term effects of dieting compared with dieting plus aerobic exercise on lactation performance. Am J Clin Nutr 1999; 69:959-67.

14. Lovelady CA, Garner KE, Moreno KL, Williams JP. The effect of weight loss in overweight lactating women on the growth of their infants. N Engl J Med 2000; 342:449-53.

15. Kac G, Benício MHD, Valente JG, Velásquez-Meléndez G. Postpartum weight retention among women in Rio de Janeiro: a follow-up study. Cad Saúde Pública 2003; 19 Suppl 1:S149-61.

16. Organização Mundial da Saúde. Estimativas das necessidades de energia e proteína de adultos e crianças. In: Junta de Conselho de Especialistas FAO/WHO/UNU, organizador. Necessidades de energia e proteínas. São Paulo: Editora Roca; 1998. p. 152. (Série de Relatos Técnicos, 724). 
17. Institute of Medicine. Nutrition during pregnancy and lactation: an implementation guide. Washington DC: National Academies Press; 1992.

18. Sichieri R. Epidemiologia da obesidade. Rio de Janeiro: EdUERJ; 1998.

19. Sichieri R, Everhart JE. Validity of Brazilian food frequency questionnaire against dietary recalls and estimated energy intake. Nutr Res 1998; 18: 1649-59.

20. Boardley DJ, Sargent RG, Coker AL, Hussey JR, Sharpe PA. The relationship between diet activity and other factors and postpartum weight change by race. Obstet Gynecol 1995; 86:834-8.

21. Hinton PS, Olson CM. Postpartum exercise and food intake: the importance of behavior-specific self-efficacy. J Am Diet Assoc 2001; 101:1430-7.

22. George GC, Hanss-Nuss H, Milani TJ, FreelandGraves JH. Food choices of low-income women during pregnancy and postpartum. J Am Diet Assoc 2005; 105:899-907.

23. Fawzi WW, Rifas-Shiman SL, Rich-Edwards JW, Willet WC, Gillman MW. Calibration of a semiquantitative food frequency questionnaire in early pregnancy. Ann Epidemiol 2004; 14:754-62.

24. Brown JE, Buzzard IM, Jacobs DR, Hannan PJ, Kushi LH, Barosso GM, et al. A food frequency questionnaire can detect pregnancy-related changes in diet. J Am Diet Assoc 1996; 96:262-6.

25. Pinheiro ABV. Tabela para avaliação de consumo alimentar em medidas caseiras. 3a Ed. Rio de Janeiro: Editora Atheneu; 1996.

26. Centro de Informática em Saúde. Programa de apoio à nutrição. São Paulo: Escola Paulista de Medicina, Universidade Federal de São Paulo; 1995.

27. Instituto Brasileiro de Geografia e Estatística. Estudo Nacional da Despesa Familiar - ENDEF. Tabela de composição de alimentos. Rio de Janeiro: Instituto Brasileiro de Geografia e Estatística; 1985.

28. Pennington JA. Food values of portions used. 15th Ed. New York: Harper \& Row Publishers; 1989.
29. Briefel RR, McDowell MA, Alaimo K, Caughman CR, Bischof A, Carrol MD, et al. Total energy intake of the US population: the third National Health and Nutrition Examination Survey 19881991. Am J Clin Nutr 1995; 62:S1072-80.

30. Krebs NF, Reidinger CJ, Hartley S, Robertson AD, Hambidge KM. Zinc supplementation during lactation: effects on maternal status and milk zinc concentrations. Am J Clin Nutr 1995; 61:1030-6.

31. Willet WC. Nutritional epidemioloy. 2nd Ed. Oxford: Oxford University Press; 1998.

32. Dewey KG, McCrory MA. Effects of dieting and physical activity on pregnancy and lactation. Am J Clin Nutr 1994; 59:S446-53.

33. Dietary Guidelines Advisory Committee. Report of the Dietary Guidelines Advisory Committee on the Dietary Guidelines for Americans 2000. Washington DC: Department of Agriculture; 2000.

34. Organización Mundial de la Salud. Dieta, nutrición y prevención de enfermedades crónicas. Geneva: Organización de las Naciones Unidas para la Agricultura y la Alimentación/Organización Mundial de la Salud; 1990. (Serie de Informes Técnicos, 797).

35. Carmo MGT, Colares LGT, Saunders C. Nutrição na lactação. In: Accioly E, Saunders C, Lacerda EMA, organizadores. Nutrição em obstetrícia e pediatria. Rio de Janeiro: Cultura Médica; 2003. p. 225-46.

36. Souza RA. Consumo de cafeína e alimentos-fonte de cafeína e prematuridade: um estudo caso-controle [Dissertação de Mestrado]. Rio de Janeiro: Instituto de Medicina Social, Universidade do Estado do Rio de Janeiro; 2004.

37. Levy-Costa RB, Sichieri R, Pontes NS, Monteiro CA. Disponibilidade domiciliar de alimentos no Brasil: distribuição e evolução (1974-2003). Rev Saúde Pública 2005; 39:530-40.

38. Sichieri R. Dietary patterns and their associations with obesity in the Brazilian city of Rio de Janeiro. Obes Res 2002; 10:42-9.

39. Sichieri R, Castro JFG, Moura AS. Fatores associados ao padrão de consumo alimentar da população brasileira urbana. Cad Saúde Pública 2003; 19 Suppl 1:S47-53.

Recebido em 06/Mai/2005

Versão final reapresentada em 12/Set/2005

Aprovado em 27/Set/2005 